\title{
Probiotic E. coli Nissle 1917 biofilms on silicone substrates for bacterial interference against pathogen colonization
}

\author{
Quan Chen, ${ }^{\ddagger}, \mathrm{a}, \mathrm{b}$ Zhiling Zhu, ${ }^{\text {,b }}$ Jun Wang, ${ }^{\mathrm{b}}$ Analette I. Lopez, ${ }^{\mathrm{b}}$ Siheng Li, ${ }^{\mathrm{b}}$ Amit \\ Kumar, ${ }^{\mathrm{b}}$ Fei Yu, ${ }^{\mathrm{b}}$ Haoqing Chen, ${ }^{\mathrm{b}}$ Chengzhi Cai ${ }^{*}$, and Lijuan Zhang ${ }^{*}$,a \\ ${ }^{a}$ School of Chemistry and Chemical Engineering, South China University of \\ Technology, Guangzhou 510640, China. \\ ${ }^{\mathrm{b}}$ Department of Chemistry, University of Houston, Houston, Texas 77204, United \\ States.
}

\begin{abstract}
Bacterial interference is an alternative strategy to fight against device-associated bacterial infections. Pursuing this strategy, a non-pathogenic bacterial biofilm is used as a live, protective barrier to fence off pathogen colonization. In this work, biofilms formed by probiotic Escherichia coli strain Nissle $1917(\mathrm{EcN})$ are investigated for their potential for long-term bacterial interference against infections associated with silicone-based urinary catheters and indwelling catheters used in the digestive system, such as feeding tubes and voice prostheses. We have shown that EcN can form stable biofilms on silicone substrates, particularly those modified with a biphenyl mannoside derivative. These biofilms greatly reduced the colonization by pathogenic Enterococcus faecalis in Lysogeny broth (LB) for 11 days.

Keywords: bacterial interference; catheter-associated urinary tract infection; E. coli Nissle 1917; mannoside; biofilms.

"Tel: +86 20 87112046; fax: +862087112046.

E-mail: cai@uh.edu (C.Cai); celjzh@scut.edu.cn (L. Zhang)
\end{abstract}

\section{Introduction}

Approximately 1.7 million patients acquire infections during treatment in hospitals and healthcare units, which is associated with approximately 99,000 deaths in the 
United States each year [1]. Most of these nosocomial infections are related to the use of indwelling medical devices, such as urinary catheters [2, 3], feeding tubes and voice prostheses $[4,5]$. Device-associated infections are mostly originated from the formation of pathogenic biofilms on the device surface, which not only shield the pathogens from host defence and antimicrobial agents but also facilitate the development of drug resistance. Antibiotics- or silver-coated urinary catheters have been clinically used to reduce pathogenic biofilm formation, but results of many clinical studies suggested that they only postponed the onset of bacteriuria less than 7 days or even ineffective in a long-term catheterization [2, 6-8]. Great progresses have recently been made in development of the next generation anti-biofilm materials and coatings exhibiting combined antifouling and antimicrobial functions [9-12]. Nevertheless, prevention of bacterial colonization after prolonged exposure to a high nutrient environment remains a great challenge and calls for the development of alternative strategies [13]. To this end, we have been pursuing an alternative strategy using non-pathogenic or probiotic bacteria that are beneficial to the hosts to form a biofilm as a live, protective barrier to withstand pathogen colonization. A unique feature of this bacterial interference strategy is that the anti-pathogen coating is live and allows for self-renewal to maintain the anti-pathogenic activity, whereas current antimicrobial coatings are subjected to the gradual covering by the biomass generated by the rapidly growing pathogens in a high nutrient environment. Among the prototype probiotics, Escherichia coli 83972 and a variety of Lactobacillus species have been reported in many clinical studies to be applied in the bacterial interference strategy against catheter-associated urinary tract infection (CAUTI) [2, 3, 14]. Recently, we reported that the biofilms of a derivative strain of E. coli 83972 prevented the colonization of a common pathogen, Enterococcus faecalis 210, grown in Lysogeny broth (LB) media at a high concentration $\left(>10^{5}\right.$ colony forming units $(\mathrm{CFU}) \mathrm{mL}^{-1}$ ) for up to 11 days [15-17]. Although probiotic or non-pathogenic microorganisms have long been used to reduce the growth of pathogenic bacteria, especially in the human gut, oral cavity and urinary tract, they are traditionally 
administered orally, or as a suspension injected to the bladder [2, 3, 14]. Few studies on bacterial interference by biofilms of non-pathogenic microorganisms on solid substrates have been reported.

The successful bacterial interference is predominately dependent upon the coverage and stability of the non-pathogenic biofilms. Unfortunately, wild-type E. coli 83972 adheres poorly to surfaces because of the lack of an intact fim operon to produce functional type 1 fimbriae as a major adhesion factor [18]. To circumvent this limitation, wild-type E. coli 83972 has been genetically modified to give strains expressing type 1 fimbriae, referred to as fim+ E. coli 83972, resulting in increased surface adherence [19], particularly the mannoside presenting surfaces through the specific binding with FimH at the tip of the fimbriae [15-17]. Although type 1 fimbriae are suggested as a virulence factor of urinary tract infection [20], one clinical study of fim + E. coli 83972 in human bladder was reported and no side effects were found [21]. Despite the encouraging results, more studies on the pathogenicity of fim+ E. coli 83972 are needed. Meanwhile, we are evaluating the bacterial interference by biofilms of the extensively studied probiotic bacterial strains, aiming to use a similar strategy to prevent pathogenic colonization on other types of indwelling medical devices in contact with commensal bacteria, such as feeding tubes and voice prostheses. To this end, we are particularly interested in the probiotic E. coli strain Nissle $1917(\mathrm{EcN})$ that has been commercialized over 90 years with the trade name "Mutaflor ${ }^{\circledR} "$ as a probiotic remedy for intestinal disorders [22]. As one of the most prevalent probiotic strains, $\mathrm{EcN}$ has been in clinical trial for treatment of ulcerative colitis [23], Crohn's disease [24] and inflammatory bowel disease due to its potentially higher capability of competing enteropathogen in the human intestine [22]. Although EcN has not been used for treatment of urinary tract infection, its presence in the urinary tract has not been reported to cause any side effects [25]. In vitro study has shown that $\mathrm{EcN}$ inhibited or out-competed most pediatric uropathogenic isolates [26]. Moreover, EcN (Mutaflor ${ }^{\circledR}$ ) is commercially available, while E. coli 83972 is not. Furthermore, EcN possesses type 1 fimbriae that greatly promote its biofilm 
formation particularly on mannoside presenting surfaces, whereas genetic modification was needed for wild-type E. coli 83972 to enhance its adherence. The above unique advantages of $\mathrm{EcN}$ prompted us to initiate this work on the investigation of the biofilm formation and bacterial interference by $\mathrm{EcN}$ on silicone as the most widely used material in biomedical devices including tracheostomy tubes [27], drainage tubes, gastro-intestinal feeding tubes and urinary catheters [28].

\section{Experimental}

\subsection{Materials}

All reagents and solutions were purchased from VWR, Becton-Dickinson, Alfa-Aesar or Sigma-Aldrich, and were used as received. Water was purified with a Milli-Q purification system (Millipore Co., Billerica, MA).

\subsection{Synthesis of mannosides}

Synthesis of the mannoside derivatives, biphenyl mannoside BiPh-Man, alkynylphenyl mannoside PPh-Man, triazolylmethyl mannoside TAM-Man, triazolylpropyl mannoside TAP-Man and oligo(ethylene glycol) mannoside Man was reported in our previous work $[15,17]$.

\subsection{Preparation of mannoside surfaces}

The mannoside surfaces were prepared following the method described in our previous publications $[15,17]$. Briefly, the poly-dimethylsiloxane (PDMS) precursors (10:1 ratio, SYLGARD 184 Silicone Elastomer Kit, Dow Corning, Midland, MI) were thoroughly mixed as instructions and allowed to stand until no air bubbles disappeared (taking about $30 \mathrm{~min}$ ). The PDMS gel was added to a clean silicon wafer (University Wafer, Boston, MA). The wafer was pressed against another silicon wafer modified with octadecyltrichlorosilane (OTS) (Sigma-Aldrich Co., St. Louis, MO). The mixture was annealed at $110{ }^{\circ} \mathrm{C}$ overnight, and the OTS-modified silicon wafer was peeled off to obtain the PDMS surface.

This surface was then treated with $\mathrm{CO}_{2}$ plasma (Harrick plasma cleaner, Model 
PDC-32G, $100 \mathrm{~W}$ ) with a $6.8 \mathrm{~W}$ low power setting for 45 seconds. The as-prepared oxidized PDMS surface was immediately immersed in a $2 \mathrm{~mL}$ generation 5 poly(amidoamine) (G5 PAMAM) dendrimer $\left(1 \mathrm{mg} \mathrm{mL}^{-1}\right.$, Dendritech, Inc., Midland, MI) in $0.01 \mathrm{M}$ phosphate buffered saline (PBS, pH = 7.42, Sigma-Aldrich Co., St. Louis, MO) for 1 hour to provide a PAMAM-modified surface. The PAMAM-modified surface was washed copiously with Milli-Q water (18.2 $\mathrm{M} \Omega \cdot \mathrm{cm} @ 25{ }^{\circ} \mathrm{C}$, Millipore Co., Billerica, MA) and dried in a flow of nitrogen (Matheson Tri-Gas, Houston, TX).

The stock solutions containing $3 \mathrm{mM}$ of the mannoside derivative (BiPh-Man, PPh-Man, TAM-Man, TAP-Man or Man, Scheme 1), 1-ethyl-3-(3-dimethylaminopropyl) carbodiimide hydrochloride (EDC, $60 \mathrm{mM}$, Alfa-Aesar, Ward Hill, MA) and N-hydroxysulfosuccinimide (sulfo-NHS, 30 mM, G-Biosciences, St. Louis, MO) in Milli-Q water were prepared. Each PAMAM substrate was immersed in $0.5 \mathrm{~mL}$ of the corresponding stock solution in separate wells of a 24-well plate for 2 hours. Subsequently the substrate was washed with copious Milli-Q water and dried with a flow of nitrogen to provide the mannoside-modified substrates. Characterization of each mannoside presenting surface was described previously [15, 17].

\subsection{Bacterial strains and preparation}

Escherichia coli strain Nissle $1917(\mathrm{EcN})$ was prepared by suspending one capsule of the Mutaflor ${ }^{\circledR}$ (Medical Futures Inc., Ontario, Canada) consisting of the bacterial strain in $50 \mathrm{~mL}$ Lysogeny broth $(\mathrm{LB}, \mathrm{pH}=7.27, \mathrm{BD}$, Franklin Lakes, NJ) and incubated overnight at $37{ }^{\circ} \mathrm{C}$ under static conditions. The resulting bacterial suspension was diluted with fresh LB at the ratio of 1:100, and re-incubated overnight at $37^{\circ} \mathrm{C}$ without shaking. The resultant bacterial suspension plated on Luria Bertani agar (BD, Franklin Lakes, NJ) after serial dilution. The plates were placed in a $37{ }^{\circ} \mathrm{C}$ incubator for 24 hours. A single colony of $\mathrm{EcN}$ from the plate was inoculated into the fresh LB broth $(50 \mathrm{~mL})$ and incubated overnight with $200 \mathrm{rpm}$ orbital shaking at 37 ${ }^{\circ} \mathrm{C}$. The concentration of the bacterial suspension was adjusted to $[\mathrm{OD}]_{600}=0.25, \sim 10^{8}$ 
$\mathrm{CFU} \mathrm{mL} \mathrm{mL}^{-1}$ for further use.

The challenge pathogenic bacteria, Enterococcus faecalis strain 210, were provided by Dr. Barbara W. Trautner (Baylor College of Medicine). The pMB158GFP plasmid was transformed into the E. faecalis to express green fluorescence protein (GFP), and that can be distinguished with fluorescence microscope. This plasmid also contain a tetracycline-resistant gene. A single colony of the E. faecalis strain 210 was inoculated into the selective $\mathrm{LB}$ media $(50 \mathrm{~mL})$ containing tetracycline $\left(4 \mu \mathrm{g} \mathrm{mL}^{-1}\right.$, Sigma-Aldrich Co., St. Louis, MO) as the antibiotic for selective culturing the GFP-carrying E. faecalis.

\subsection{Preparation of EcN biofilms}

The mannoside-presenting substrates and control (PDMS and G5 PAMAM) substrates were cut into pieces of $1 \times 1 \mathrm{~cm}^{2}$. The corresponding sample was separately placed in a 24 -well plate and inoculated with $\mathrm{EcN}$ bacterial suspension $\left(1 \mathrm{~mL}, 10^{8}\right.$ $\left.\mathrm{CFU} \mathrm{mL}{ }^{-1},[\mathrm{OD}]_{600}=0.25\right)$. The substrates were incubated without shaking at $37^{\circ} \mathrm{C}$ for 5 days for the formation of $\mathrm{EcN}$ biofilms, during which the same $\mathrm{EcN}$ culture was changed daily. Afterwards, the substrates rinsed with 0.01 M PBS solution three times to remove the planktonic EcN prior to microscopic imaging.

\subsection{Stability of EcN biofilm on silicone under shaking conditions}

Freshly prepared EcN biofilms on silicone substrates with/without PAMAM and BiPh-Man modification were placed in round bottom tubes (BD Falcon, $14 \mathrm{~mL}$ Polystyrene Round-Bottom Tube) and immersed with $1 \mathrm{~mL}$ artificial urine (AU, $\mathrm{pH}=$ 7.04) [29]. The tubes were shaken in the orbital shaker at $3 / 4$ inch circular orbit and $200 \mathrm{rpm}$ for $10 \mathrm{~min}$. The detached $\mathrm{EcN}$ in $\mathrm{AU}$ was quantified by plate counting. The procedure was repeated two additional times. Each silicone substrate was washed with AU three times by gently shaken and refilled with $1 \mathrm{~mL}$ AU. Finally, the bacteria remaining on the substrate surface were fully detached for plate counting by sonication using ultrasonic cleaner (B-12, Branson, Danbury, CT) at $80 \mathrm{~W}, 20^{\circ} \mathrm{C}$ for $10 \mathrm{~min}$, followed by vortexing for $1.5 \mathrm{~min}$ (2000 rpm, Mini Vortexter MV 1, VWR 
Scientific Products, Radnor, PA) in $1 \mathrm{~mL} 0.01 \%$ sodium dodecyl sulfate (SDS, J.T.Baker Inc. Phillipsburg, NJ) in 0.01 M PBS solution. Serial dilutions of the resultant bacterial suspension were prepared, as $10^{0}, 10^{-2}, 10^{-4}, 10^{-6}$ and $10^{-8}$, respectively. $100 \mu \mathrm{L}$ of each suspension was plated on Luria Bertani agar in duplicates and incubation at $37^{\circ} \mathrm{C}$ for 24 hours. The bacteria colonies formed on the plates were counted.

\subsection{Bacterial interference against E. faecalis colonization}

The substrates pre-coated with biofilms of EcN were separately placed in a 24-well plate and immersed with E. faecalis strain 210 suspension $\left(10^{8} \mathrm{CFU} \mathrm{mL}^{-1},[\mathrm{OD}]_{600}=\right.$ 0.25 ) in $1 \mathrm{~mL} \mathrm{LB}$ media (without antibiotics). The plate was placed in a $37{ }^{\circ} \mathrm{C}$ incubator under static conditions and incubated for 11 days in the above E. faecalis culture. During this period, the bacterial culture was changed daily. After 11 days bacterial interference, each sample was taken out from the bacterial culture, and immersed in a petri dish $\left(60 \times 15 \mathrm{~mm}^{2}\right.$, Sigma-Aldrich) half-filled with $1 \mathrm{~mL} 0.01 \mathrm{M}$ PBS. The dish was gently shaken three times in back and forth motions on top of a desk by hand. This gentle washing steps were repeated three times to detach the weakly bounded planktonic bacteria prior to the microscopy examination. For a control, the corresponding mannoside presenting surfaces without pre-treatment with EcN were subjected to the above treatment with E. faecalis strain 210.

\subsection{Microscopy imaging of the EcN biofilms}

All images from the bacterial adherence and interference assays were acquired with a $40 \times$ objective in the reflected bright-field or with FITC filters in a Nikon $80 \mathrm{i}$ Microscope (Nikon Instruments, Melville, NY). A CoolSnap HQ2 camera (Photometrics, Tucson, AZ) and NIS Elements software (Version 3.0, Nikon Instruments, Melville, NY) were used for image acquisition and analysis. In the bacterial interference assay, E. faecalis was visualized with FITC channel and the $\mathrm{EcN}$ is non-fluorescent. The fluorescence and the corresponding reflected bright-field images were recorded on ten random locations for each sample, and the experiments 
were repeated at least three times. For the non-pathogenic biofilms challenged by the E. faecalis strain 210 expressing GFP, the coverage by both bacteria on a reflected bright-field image was obtained, as well as the coverage by the green fluorescent $E$. faecalis in FITC channel over the same area. The coverage of E. faecalis was acquired using the NIS Elements Software on the fluorescence images, which represent an underestimate for high coverage E. faecalis and $\mathrm{EcN}$ films where bacteria above and underneath the focal plane were not counted. The EcN coverage was calculated by subtracting the E. faecalis coverage and it is possible that the presence of EcN under E. faecalis strain 210 was ignored, resulted in an underestimate of the $\mathrm{EcN}$ coverage.

\subsection{Z-series acquisition (Z-scan)}

The reflected bright-field Z-scan images (step: $1.5 \mu \mathrm{m}$, range $\mathrm{z}_{0}: \pm 15.0 \mu \mathrm{m}$ ) were acquired by a $40 \times$ objective on the $\mathrm{EcN}$ biofilm formed on a BiPh-Man presenting surface after $48 \mathrm{~h}$ incubation and 18 hour of $2.5 \%$ glutaraldehyde (Sigma-Aldrich Co., St. Louis, MO) fixation in $0.01 \mathrm{M}$ PBS at $4{ }^{\circ} \mathrm{C}$. The CoolSnap HQ2 camera and NIS Elements software were employed for image acquisition and analysis.

\subsection{Quantification of bacterial interference by plate counting}

For selective plate counting of EcN and E. faecalis 210 in a mixture, the resistance of EcN against tetracycline was first established. Luria Bertani agar with chloramphenicol (20 $\mu \mathrm{g} \mathrm{mL}^{-1}$, Sigma-Aldrich), tetracycline $\left(4 \mu \mathrm{g} \mathrm{mL} \mathrm{m}^{-1}\right.$, Sigma-Aldrich) or ampicillin (100 $\mu \mathrm{g} \mathrm{mL}^{-1}$, Sigma-Aldrich) were tested, and the non-antibiotic LB agar was used as a control. Culture of E. faecalis 210 and $\mathrm{EcN}$ were aforementioned, centrifuged at $4800 \mathrm{rpm}$ for $10 \mathrm{~min}$ and washed with $0.01 \mathrm{M}$ PBS. Subsequently, the pellet was resuspended with $0.01 \mathrm{M} \mathrm{PBS}$ and adjusted to $[\mathrm{OD}]_{600}=$ 0.25 , and diluted by the factors of $10^{2}, 10^{4}, 10^{6}$ and $10^{8}$, respectively. $100 \mu \mathrm{L}$ of each diluted suspension was plated in duplicates on a LB agar plate with the above antibiotics and the non-antibiotic control. The plates were placed into a $37{ }^{\circ} \mathrm{C}$ incubator for 24 hours and counted the formed bacterial, as colonies colony forming unit per $\mathrm{mL}\left(\mathrm{CFU} \mathrm{mL} \mathrm{m}^{-1}\right)$. The results indicated that E. faecalis 210 could grow on the 
agar only plate $\left(\mathrm{CFU}=2.0 \times 10^{8}\right)$ or the tetracycline agar plate $\left(\mathrm{CFU}=1.9 \times 10^{8}\right)$, while $\mathrm{EcN}$ could grow on the agar only plate $\left(\mathrm{CFU}=2.0 \times 10^{8}\right)$ but could not survive on the tetracycline agar plate $\left(\mathrm{CFU}<10^{2}\right)$. Therefore, tetracycline was chosen for the selective culture of E. faecalis 210 strain.

After 11 days bacterial interference, the samples were rinsed with $1 \mathrm{~mL} 0.01 \mathrm{M}$ PBS and gently transferred to a flat bottom vial containing $1 \mathrm{~mL}$ solution of $0.01 \%$ sodium dodecyl sulfate (SDS) solution, and sonicated for $10 \mathrm{~min}$ followed by vortexing for another $1.5 \mathrm{~min}$. Serial dilutions from the sonicated bacterial suspension were prepared as $10^{0}, 10^{-2}, 10^{-4}, 10^{-6}$ and $10^{-8} .100 \mu \mathrm{L}$ of each dilution was plated in triplicates on the $\mathrm{LB}$ agar with tetracycline $\left(4 \mu \mathrm{g} \mathrm{mL}^{-1}\right)$. The plates were placed into a $37^{\circ} \mathrm{C}$ incubator for 24 hours to generate colonies. Finally, the E. faecalis 210 colonies were counted and expressed as CFU $\mathrm{mL}^{-1}$.

\subsection{Viability of EcN after subjected to sonication and vortex}

$\mathrm{EcN}$ was prepared as aforementioned, centrifuged (5000 rpm, $10 \mathrm{~min}$ ) and rinsed with $0.01 \mathrm{M}$ PBS three times. The EcN pellet was adjusted to $\mathrm{OD}_{600}=0.25$ by addition of $0.01 \mathrm{M}$ PBS or $0.01 \%$ SDS solution. Afterwards, the suspension was sonicated for $1 \mathrm{~min}$ and $10 \mathrm{~min}$, followed by vortexing for $1.5 \mathrm{~min}$. Suspensions in 0.01 M PBS without sonication or vortexing were used as the control groups. All the samples were diluted by factors of $10^{2}, 10^{4}, 10^{6}$ and $10^{8} .100 \mu \mathrm{L}$ of each diluted suspension was plated in duplicates on the LB agar. The plates were placed in $37^{\circ} \mathrm{C}$ incubator for 24 hours to generate the bacterial colonies. Consequently, the EcN

colonies were counted and expressed as $\mathrm{CFU} \mathrm{mL} \mathrm{m}^{-1}$. The results were summarized in Fig. S3.

\subsection{Scanning electron microscopy (SEM)}

The immobilized bacteria were fixed with $2.5 \%$ glutaraldehyde (Sigma-Aldrich Co., St. Louis, MO) in $0.01 \mathrm{M}$ PBS for 18 hours at $4{ }^{\circ} \mathrm{C}$, and then washed with $0.01 \mathrm{M}$ PBS. The sample surfaces were stained with $1 \% \mathrm{OsO}_{4}$ (Electron Microscopy Sciences, Hatfield, PA) in $1 \mathrm{~mL} 0.01 \mathrm{M}$ PBS for 2 hours at room temperature, rinsed with 0.01 
M PBS, and dehydrated in an ascending ethanol series $(25 \%, 50 \%, 70 \%, 85 \%, 95 \%$ and $100 \%$ ) for $10 \mathrm{~min}$ each. Finally, the samples were immersed in $t$-butanol for 20 min, followed by freeze-drying using a Scanvac CoolSafe 110-4 (LaboGene). The freeze-dried samples were then sputter-coated with gold (Hummer 6.2 Sputter System, Anatech, USA) and examined by SEM (LEO, model 1525) at an accelerating voltage of $15 \mathrm{kV}$.

\subsection{Statistical analysis}

All data were obtained from three repeated experiments and expressed as mean \pm standard deviation. Statistical analysis between groups were performed by Students' $t$ test. Statistical differences were determined by Tukey's multiple comparison test. A $P$ value $<0.05$ was considered to be statistically significant.

\section{Results and discussion}

\subsection{Formation of the EcN biofilms on silicone surfaces}

Silicone substrates were modified with mannosides as previously described, involving surface activation with $\mathrm{CO}_{2}$ plasma, immobilization of the generation 5 poly(amidoamine) (G5 PAMAM) dendrimers, followed by attachment of a series of mannoside derivatives with a $\mathrm{COOH}$ handle (Scheme 1) via amidation $[15,17]$. These mannoside derivatives differ in glycosidic linkages, including biphenyl (BiPh), propylphenyl (PPh) and triazolylalkyl (TAM and TAP, Scheme 1), which impose a wide range of binding affinities to the FimH receptor at the tip of the type 1 fimbriae [30-32].

To promote type 1 fimbriae expression in the bacteria, the preparation of $\mathrm{EcN}$ biofilm on the above mannoside presenting surfaces was conducted in static conditions (see details in Experimental section) [22, 33]. In compared with fim $+E$. coli 83972 that formed biofilms on mannoside surfaces after incubation for 2 days [15, 17], EcN took 5 days to form biofilms on the high affinity mannoside surfaces. The slower growth rate for $\mathrm{EcN}$ might be due to the phase switching that turned on or off the expression of type 1 fimbriae $[22,33]$. 
The reflected bright-field images in Fig. 1 and data summarized in Fig. 2 show that the biofilm coverage of $\mathrm{EcN}$ was sensitive to the surface mannosides varying in the glycosidic linkage. The biofilm coverage on the BiPh-Man surfaces was 4-fold higher than on the unmodified PDMS surface. In comparison, the coverage of $f i m+E$. coli 83972 biofilms were affected less by the mannoside surfaces, with a 2.5 -fold increase on the BiPh-Man surface [17]. A similar biofilm coverage was observed on the control surfaces (unmodified PDMS and G5 PAMAM) versus on the surface presenting TAM-Man with a short alkyl linker (Scheme 1), which was only half of that on the Man surface. The surfaces of TAP-Man, alkynylphenyl mannoside PPh-Man and biphenyl mannoside BiPh-Man greatly increased the biofilm coverage. The overall tendency followed the order of BiPh-Man > PPh-Man > TAP-Man > Man > TAM-Man $\approx$ G5 PAMAM $\approx$ PDMS, similar to the one for fim+ E. coli 83972 and corresponding to the relative binding affinity of each mannoside to the FimH adhesin at the tip of type 1 fimbriae [30-32].

SEM imaging of a fim + E. coli 83972 biofilm grown on the biphenylmannoside BiPh-Man surface for 5 days revealed the presence of lamellar and chunk extracellular polymeric substances (EPS, Fig. 3). Most single or stacks of bacteria were connected by these EPS to form a cross-linked net structure. Wide-field optical microscopy imaging showed the presence of apertures and holes $(<100 \mathrm{~nm}$, Fig. 1g, $3 b$ and 3c) that were presumably the entrance of channels [34] for transporting nutrients and wastes in and out of the biofilm. The average thickness of the densely packed biofilms was about $10 \mu \mathrm{m}$, corresponding to about ten stacked bacterial cell layers, as shown in the SEM images of the cross section of biofilm, e.g. Fig. 3c. The thickness of the biofilms was also estimated to be about $15 \mu \mathrm{m}$ with optical microscopy using z-scan technique (Fig. 4) [35].

\subsection{Stability of EcN biofilms on silicone surfaces}

The mechanical stability of the EcN biofilms grown on the biphenylmannoside BiPh-Man surface was compared with those grown on the unmodified PDMS and PAMAM surfaces. Specifically, the samples fully immersed in $1 \mathrm{~mL}$ artificial urine 
(AU) was shaken at 3/4 inch circular orbit at $200 \mathrm{rpm}$ for $10-30 \mathrm{~min}$ to generate a periodic shear stress with a magnitude of 0.5-2 $\mathrm{Pa}$ [36], which was higher than the maximum shear stress $(0.5 \mathrm{~Pa})$ on the ureter [37]. The bacteria remained on the surface were detached by mild ultrasound and plate-counted. The results showed that the BiPh-Man modified silicone substrate retained the most bacteria after $30 \mathrm{~min}$ of shaking (Table 1). On the other hand, there were approximately $60 \%$ bacteria detached from the unmodified PDMS or G5 PAMAM modified silicone substrates. The results illustrate the establishment of a strong binding between the EcN biofilms and the BiPh-Man modified silicone substrate, which could withstand a shear stress similar to the urine flow.

\subsection{Bacterial interference against E. faecalis colonization}

To examine the performance of the bacterial interference against pathogen colonization, we used the Gram-positive multidrug-resistant Enterococcus faecalis strain 210 [38] to continuously challenge the above biofilms of $\mathrm{EcN}$ on various mannoside presenting surfaces. This pathogen was chosen since E. faecalis have a strong ability to form biofilms [39], and are among the organisms causing the most challenging hospital acquired infections [40]. The substrates were incubated with the competing bacterial suspension $\left(10^{8} \mathrm{CFU} \mathrm{mL}^{-1}\right)$ in $1 \mathrm{~mL} \mathrm{LB}$ media for up to 11 days. The culture was changed with new suspension of E. faecalis suspension $\left(10^{8} \mathrm{CFU}\right.$ $\mathrm{mL}^{-1}$ ) in LB every $24 \mathrm{~h}$. After culturing, for 11days, the culture was removed. The samples were taken out, and gently washed three times with 0.01 M PBS before being examining by a fluorescence microscope and plate-counting. It should be emphasized that the gentle washing procedure described in the experimental section was used to detach only the planktonic bacteria, although it might not prevent some leftover of $E$.

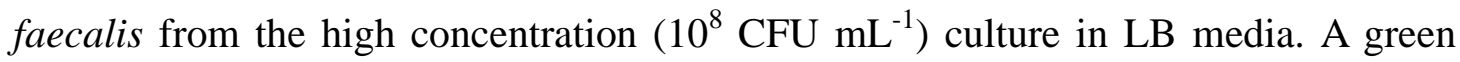
fluorescent protein (GFP) modified E. faecalis strain was used in this study to distinguish from the non-fluorescent $\mathrm{EcN}$ strain by fluorescence microscope. The substrates without the pre-established non-pathogenic biofilms were treated as the control. 
Fig. 5a plots the coverage of E. faecalis measured by their green fluorescence on various surfaces. The colonization of E. faecalis to all surfaces without pre-formed non-pathogenic biofilms exhibited no significant difference $(* P=0.641$, Tukey's test), as the fully covered E. faecalis biofilms (Fig. 6h and S1). On the other hand, after long-term (11 days) exposure to the E. faecalis culture at a high concentration $\left(10^{8} \mathrm{CFU} \mathrm{mL} \mathrm{m}^{-1}\right)$, pre-coating with $\mathrm{EcN}$ biofilms reduced the adherence of the $E$. faecalis on all surfaces including unmodified PDMS and G5 PAMAM modified surfaces (Fig. 5a, 6a-g and S2). In these two surfaces, pre-coating with fim+ E. coli 83972 did not prevent E. faecalis colonization after 11 days [16]. However, pre-coating with EcN did substantially reduce the E. faecalis colonization (Fig. 5a). Furthermore, as shown by Fig. 5a, fewer E. faecalis adherence on all surfaces pre-coated with EcN as compared with fim+ E. coli $83972[20](* P=0.016, t$ test $)$. The bacterial interference performance by the $\mathrm{EcN}$ biofilm on different mannoside presenting surfaces as well as control surfaces follows the order of BiPh-Man > $\mathbf{P P h}-$ Man $>$ TAP-Man $\approx$ PDMS > G5 PAMAM > Man $\approx$ TAM-Man.

Besides microscopic imaging that revealed the spatial distribution of E. faecalis on the $\mathrm{EcN}$ biofilms and provided a rough estimate of their amounts, we also performed selective plate-counting of the adherent $E$. faecalis using tetracycline $\left(4 \mu \mathrm{g} \mathrm{mL}^{-1}\right)$ as the antibiotic [41]. The bacteria were detached by sonication and vortexed in a $0.01 \%$ sodium dodecyl sulfate (SDS) solution. This procedure was validated that the bacteria remained viable under the condition. As shown in Fig. 5a and 5b, the microscopic estimation and plate counting results point to the same ranking (see above) of the mannoside and control surfaces for the efficiency of bacterial interference by the $\mathrm{EcN}$ biofilms grown on these surfaces against $E$. faecalis colonization.

The above result of the bacterial interference assay was similar to the order for initial biofilm coverage of the $\mathrm{EcN}$ on the mannoside-presenting surfaces. A closer look at the magnified overlay of the reflected bright-field and fluorescence images in Fig. 6d revealed that a dense, stable non-pathogenic biofilm of $\mathrm{EcN}$ was remained on the BiPh-Man presenting surface even after an 11 days continuous challenge with $E$. 


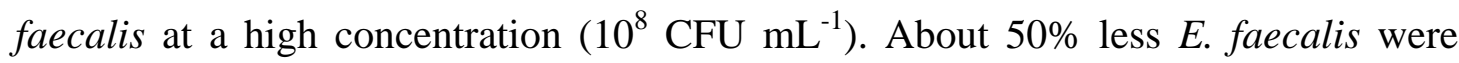
found on the EcN biofilms than on the fim + E. coli 83972 biofilms [17]. Furthermore, we found that unlike fim + E. coli 83972 biofilms [17], the EcN biofilms formed on the unmodified PDMS surfaces performed equally well as those modified by the mannosides Man, TAP-Man and TAM-Man with a relatively low affinity to FimH. This result shows that EcN biofilms on the unmodified PDMS surfaces were more efficient for prolonged bacterial interference than fim+ E. coli 83972 biofilms.

\subsection{EcN biofilm stability after long-term bacterial interference}

After the bacterial interference assay for 11 days, in comparison to the fim+ E. coli 83972 biofilm [17], the biofilm of $\mathrm{EcN}$ maintained a higher coverage on these mannoside-modified PDMS surfaces (Fig. S4). After challenged by E. faecalis for up to 11 days, the EcN biofilm coverage and stability was in the order of Bi-Man > PPh-Man > TAP-Man > Man > TAM-Man $\approx$ G5 PAMAM $\approx$ PDMS, as shown in Fig. S4, which was similar to the order of the EcN biofilm formation. Remarkably, the coverage of the biofilms of EcN on the BiPh-Man and PPh-Man surfaces maintained high, being $88 \%$ and $75 \%$, respectively. This result closely correlates with the high efficiency of the EcN biofilms grown on theses mannoside surfaces for preventing $E$. faecalis colonization.

\section{Conclusions}

In conclusion, we have demonstrated the first use of pre-established $\mathrm{EcN}$ biofilms on silicone to largely reduce pathogenic colonization by E. faecalis 210 . We showed that EcN biofilms performed better than fim + E. coli 83972 biofilms for prolonged bacterial interference against pathogen colonization. The best result was obtained from EcN biofilms formed on BiPh-Man modified PDMS surfaces. We note that in comparison to many other reports on surface modification against pathogenic bacterial adhesion, our results were obtained in a high-nutrient environment (LB media) [13] with aggressive biofilm-forming pathogen E. faecalis 210 at a 
concentration of $10^{8} \mathrm{CFU} \mathrm{mL}^{-1}$ that is 100,000 times above the diagnostic threshold of urinary tract infection [42] for up to 11 days. Under such conditions, the biomass generated by the rapidly growing bacteria could foul the surfaces modified by other strategies. Our result suggested that EcN biofilms pre-coated on silicone surfaces may serve as a living prophylactic agent that effectively outcompetes pathogens against their colonization and biofilm formation. Moreover, $\mathrm{EcN}$ in oral administrable capsule is commercially available, and has been extensively studied. Our results encourage further investigation of the bacterial interference strategy using $\mathrm{EcN}$ biofilms coated on indwelling medical devices for potential use in the gas intestinal tract and the lower urinary track. The investigation should address safety concerns particularly for use in urinary track, the risk of acquiring virulence genes from pathogens over long term, as well as the mechanism for bacterial interference.

\section{Acknowledgements}

We thank Dr. Barabara W. Trautner for providing E. faecalis 210. This study was supported by the National Natural Science Foundation of China (91434125), National Science Foundation grant (DMR-1207583) and the National Institute of Health grant (HD058985), Science and Technology Foundation of Guangdong Province, China (2015B020237008, 2016B020242004).

\section{Appendix A. Supplementary data}

Supplementary data associated with this article can be found, in the online version, at “http://www.journals.elsevier.com/Acta Biomaterialia."

I Q. Chen and Z. Zhu contributed equally to this work.

\section{References}

[1] A. Y. Peleg and D. C. Hooper, Hospital-acquired infections due to gram-negative bacteria. New. Engl. J. Med. 362 (2010) 1804-1813. 
[2] D. M. Siddiq and R. O. Darouiche, New strategies to prevent catheter-associated urinary tract infections. Nat. Rev. Urol. 9 (2012) 305-314.

[3] R. O. Darouiche and R. A. Hull, Bacterial interference for prevention of urinary tract infection. Clin. Infect. Dis. 55 (2012) 1400-1407.

[4] M. J. Talpaert, A. Balfour, S. Stevens, M. Baker, F. A. Muhlschlegel and C.

W. Gourlay, Candida biofilm formation on voice prostheses. J. Med. Microbiol. 64 (2015) 199-208.

[5] K. Bertl, V. Zijnge, B. Zatorska, M. Leonhard, B. Schneider-Stickler and H. J. M. Harmsen, Oral cavity anaerobic pathogens in biofilm formation on voice prostheses. Head \& Neck 37 (2015) 524-529.

[6] C. Desrousseaux, V. Sautou, S. Descamps, O. P. Traoré, Modification of the surfaces of medical devices to prevent microbial adhesion and biofilm formation. J. Hosp. Infect. 85 (2013) 87-93.

[7] R. Pickard, T. Lam, G. MacLennan, K. Starr, M. Kilonzo, G. McPherson, K. Gillies, A. McDonald, K. Walton, B. Buckley, C. Glazener, C. Boachie, J. Burr, J. Norrie, L. Vale, A. Grant, J. N'Dow, Antimicrobial catheters for reducing symptomatic urinary tract infections in adults requiring short-term catheterisation in hospital: a multicentre randomised controlled trial. Lancet 380 (2012) 1927-1935.

[8] D. G. Desai, K. S. Liao, M. E. Cevallos, B. Trautner, Silver or nitrofurazone impregnation of urinary catheters has a minimal effect on uropathogen adherence. J. Urology 184 (2010) 2565-2571.

[9] D. Campoccia, L. Montanaro and C. R. Arciola, A review of the biomaterials technologies for infection-resistant surfaces. Biomaterials 34 (2013) 8533-8554.

[10] B. R. Coad, H. J. Griesser, A. Y. Peleg, A. Traven, Anti-infective surface coatings: design and therapeutic promise against device-associated infections. Plos Pathog. 12 (2016) e1005598. 
[11] B. Cao, L. L. Li, Q. Tang and G. Cheng, The impact of structure on elasticity, switchability, stability and functionality of an all-in-one carboxybetaine elastomer. Biomaterials 34 (2013) 7592-7600.

[12] G. A. Junter, P. Thébault, L. Lebrun, Polysaccharide-based antibiofilm surfaces. Acta Biomater. 30 (2016) 13-25.

[13]J. A. Lichter, K. J. Van Vliet and M. F. Rubner, Design of antibacterial surfaces and interfaces: polyelectrolyte multilayers as a multifunctional platform. Macromolecules 42 (2009) 8573-8586.

[14] G. Reid, J. A. Younes, H. C. Van der Mei, G. B. Gloor, R. Knight and H. J. Busscher, Microbiota restoration: natural and supplemented recovery of human microbial communities. Nat. Rev. Micro. 9 (2011) 27-38.

[15] A. I. Lopez, A. Kumar, M. R. Planas, Y. Li, T. V. Nguyen and C. Cai, Biofunctionalization of silicone polymers using poly(amidoamine) dendrimers and a mannose derivative for prolonged interference against pathogen colonization. Biomaterials 32 (2011) 4336-4346.

[16] B. W. Trautner, A. I. Lopez, A. Kumar, D. M. Siddiq, K. S. Liao, Y. Li, D. J. Tweardy and C. Cai, Nanoscale surface modification favors benign biofilm formation and impedes adherence by pathogens. Nanomedicine: NBM 8 (2012) 261-270.

[17] Z. Zhu, J. Wang, A. Lopez, F. Yu, Y. Huang, A. Kumar, S. Li, L. Zhang and C. Cai, Surfaces presenting $\alpha$-phenyl mannoside derivatives enable formation of stable, high coverage, non-pathogenic Escherichia coli biofilms against pathogen colonization. Biomater. Sci. 3 (2015) 842-851.

[18] P. Klemm, V. Roos, G. C. Ulett, C. Svanborg and M. A. Schembri, Molecular characterization of the Escherichia coli asymptomatic bacteriuria strain 83972: the taming of a pathogen. Infect. Immun. 74 (2006) 781-785.

[19] B. W. Trautner, M. E. Cevallos, H. Li, S. Riosa, R. A. Hull, S. I. Hull, D. J. Tweardy and R. O. Darouiche, Increased expression of type-1 fimbriae by 
nonpathogenic Escherichia coli 83972 results in an increased capacity for catheter adherence and bacterial interference. J. Infect. Dis. 198 (2008) 899-906.

[20] I. Connell, W. Agace, P. Klemm, M. Schembri, S. Mărild and C. Svanborg, Type 1 fimbrial expression enhances Escherichia coli virulence for the urinary tract. Proc. Nat. Acad. Sci. 93 (1996) 9827-9832.

[21] G. Bergsten, B. Wullt, M. A. Schembri, I. Leijonhufvud and C. Svanborg, Do type 1 fimbriae promote inflammation in the human urinary tract? Cell. Microbiol. 9 (2007) 1766-1781.

[22] V. Hancock, M. Dahl and P. Klemm, Probiotic Escherichia coli strain Nissle 1917 outcompetes intestinal pathogens during biofilm formation. J. Med. Microbiol. 59 (2010) 392-399.

[23] B. J. Rembacken, A. M. Snelling, P. M. Hawkey, D. M. Chalmers and A. T. R. Axon, Non-pathogenic Escherichia coli versus mesalazine for the treatment of ulcerative colitis: a randomised trial. Lancet 354 (1999) 635-639.

[24] H. A. Malchow, A new approach in therapy to maintain remission of colonic Crohn's disease? J. Clin. Gastroenterol. 25 (1997) 653-658.

[25] G. W. Tannock, I. S. Tiong, P. Priest, K. Munro, C. Taylor, A. Richardson and M. Schultz, Testing probiotic strain Escherichia coli Nissle 1917 (Mutaflor) for its ability to reduce carriage of multidrug-resistant $E$. coli by elderly residents in long-term care facilities. J. Med. Microbiol. 60 (2011) 366-370.

[26] D. W. Storm, S. A. Koff, D. J. Horvath, Jr., B. Li and S. S. Justice, In vitro analysis of the bactericidal activity of Escherichia coli Nissle 1917 against pediatric uropathogens. J. Urol. 186 (2011) 1678-1683.

[27] P. Kaali, D. Momcilovic, A. Markström, R. Aune, G. Czel and S. Karlsson, Degradation of biomedical polydimethylsiloxanes during exposure to in vivo biofilm environment monitored by FE-SEM, ATR-FTIR, and MALDI-TOF MS. J. App. Polylm. Sci. 115 (2010) 802-810.

[28] R. Yoda, Elastomers for biomedical applications. J. Biomater. Sci.-Polym. Ed. 9 (1998) 561-626. 
[29] Z. Han, J. S. Pinkner, B. Ford, R. Obermann, W. Nolan, S. A. Wildman, D. Hobbs, T. Ellenberger, C. K. Cusumano, S. J. Hultgren and J. W. Janetka, Structure-based drug design and optimization of mannoside bacterial fimH antagonists. J. Med. Chem. 53 (2010) 4779-4792.

[30] T. Klein, D. Abgottspon, M. Wittwer, S. Rabbani, J. Herold, X. Jiang, S. Kleeb, C. Lüthi, M. Scharenberg, J. Bezençon, E. Gubler, L. Pang, M. Smiesko, B. Cutting, O. Schwardt and B. Ernst, FimH antagonists for the oral treatment of urinary tract infections: from design and synthesis to in vitro and in vivo evaluation. J. Med. Chem. 53 (2010) 8627-8641.

[31] J. Bouckaert, J. Mackenzie, J. L. de Paz, B. Chipwaza, D. Choudhury, A. Zavialov, K. Mannerstedt, J. Anderson, D. Pierard, L. Wyns, P. H. Seeberger, S. Oscarson, H. De Greve and S. D. Knight, The affinity of the FimH fimbrial adhesin is receptor-driven and quasi-independent of Escherichia coli pathotypes. Mol. Microbiol. 61 (2006) 1556-1568.

[32] M. Touaibia, A. Wellens, T. C. Shiao, Q. Wang, S. Sirois, J. Bouckaert and R. Roy, Dendrimers with nanomolar affinities to Escherichia coli FimH. ChemMedChem 2 (2007) 1190-1201.

[33] B. Stentebjerg-Olesen, T. Chakraborty and P. Klemm, Type 1 fimbriation and phase switching in a natural Escherichia coli FimB null strain Nissle 1917. J. Bacteriol. 181 (1999) 7470-7478.

[34] P. S. Stewart, Convection around biofilms. Biofouling 28 (2012) 187-198.

[35] M. Sheik-bahae, A. A. Said and E. W. Van Stryland, High-sensitivity, single-beam $n_{2}$ measurements. Opt. Lett. 14 (1989) 955-957.

[36] M. M. Salek, P. Sattari and R. J. Martinuzzi, Analysis of fluid flow and wall shear stress patterns inside partially filled agitated culture well plates. Ann. Biomed. Eng. 40 (2012) 707-728.

[37] B. Vahidi, N. Fatouraee, A. Imanparast and A. N. Moghadam, Simulation of the ureter: effects of the model parameters on ureteral pressure/flow relations. J. Biomech. Eng.-T ASME 133 (2011) 031004-1-8. 
[38] B. W. Trautner, R. O. Darouiche, R. A. Hull, S. Hull and J. I. Thornby, Pre-inoculation of urinary catheters with Escherichia coli 83972 inhibits catheter colonization by Enterococcus faecalis. J. Urol. 167 (2002) 375-379.

[39] A. Bridier, F. Dubois-Brissonnet, A. Boubetra, V. Thomas and R. Briandet, The biofilm architecture of sixty opportunistic pathogens deciphered using a high throughput CLSM method. J. Microbiol. Methods 82 (2010) 64-70.

[40] C. A. Arias and B. E. Murray, The rise of the Enterococcus: beyond vancomycin resistance. Nat. Rev. Micro. 10 (2012) 266-278.

[41] S. Kleta, M. Nordhoff, K. Tedin, L. H. Wieler, R. Kolenda, S. Oswald, T. A. Oelschlaeger, W. Bleiss and P. Schieracka, Role of F1C fimbriae, flagella, and secreted bacterial components in the inhibitory effect of probiotic Escherichia coli Nissle 1917 on atypical enteropathogenic E. coli infection. Infect. Immun. 82 (2014) 1801-1812.

[42] O. Khantamat, C.-H. Li, F. Yu, A.C. Jamison, W.-C. Shih, C. Cai, T.R. Lee, Gold nanoshell-decorated silicone surfaces for the near-infrared (NIR) photothermal destruction of the pathogenic bacterium E. faecalis. ACS Appl. Mater. Inter. 7 (2015) 3981-3993. 
Table 1. Counts of $\mathrm{EcN}$ detached into artificial urine (AU, $1 \mathrm{~mL}$ ) and remained on unmodified PDMS silicone, PAMAM coated silicone and BiPh-Man coated silicone after shaking for 10-30 $\mathrm{min}$.

\begin{tabular}{|c|c|c|c|c|c|c|}
\hline \multirow{2}{*}{ Substrate } & \multirow{2}{*}{$\begin{array}{c}\text { Initial bacteria } \\
\text { on surface } \\
\left(10^{5} \mathrm{CFU}\right. \\
\left.\mathrm{cm}^{-2}\right)\end{array}$} & \multicolumn{3}{|c|}{ 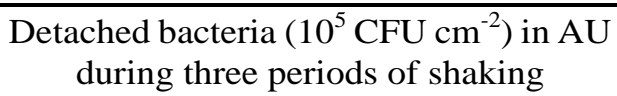 } & \multirow{2}{*}{$\begin{array}{c}\text { Bacterial } \\
\text { remained * } \\
\left(10^{5} \mathrm{CFU}\right. \\
\left.\mathrm{cm}^{-2}\right)\end{array}$} & \multirow{2}{*}{$\begin{array}{c}\% \\
\text { bacteria } \\
\text { remained } \\
*\end{array}$} \\
\hline & & $0-10 \mathrm{~min}$ & $10-20 \mathrm{~min}$ & $20-30 \mathrm{~min}$ & & \\
\hline & & & & & & 44 \\
\hline & & & & & & \\
\hline BiPh-Man & $300 \pm 90$ & $22.5 \pm 6.7$ & $2.2 \pm 0.4$ & $0.98 \pm 0.13$ & $274 \pm 83$ & 91 \\
\hline
\end{tabular}

* Bacteria remained on the substrates were counted after shaking for $30 \mathrm{~min}$. 


\section{Figure captions:}

Scheme 1 Modification of PDMS with various mannosides for evaluation of their effectiveness in promoting the growth of non-pathogenic E. coli Nissle 1917 biofilms for bacterial interference against pathogenic colonization. The mannosides BiPh-Man, PPh-Man, TAM-Man, TAP-Man, and Man are differed by the glycosidic linkage $\mathrm{R}$ as shown in the box.

Fig. 1. Representative reflected bright-field images of $\mathrm{EcN}$ on control and mannoside-presenting PDMS surfaces after 5 days adhesion. Each surface was incubated with $1 \mathrm{~mL} \mathrm{LB}$ containing $10^{8} \mathrm{CFU} \mathrm{mL}^{-1}\left([\mathrm{OD}]_{600}=0.25\right) \mathrm{EcN}$. The representative reflected bright-field image of EcN on BiPh-Man surface shows a complete coverage of the surface. In the zoom-in image, the dark spots on the relatively flat biofilm surface are presumably the entrance of channels $[4,5]$ for transporting nutrients and wastes in and out of the biofilm. Each image is representative of up to 10 images obtained on random locations at each surface and 3 experiments were repeated.

Fig. 2. Plot of the coverage of $\mathrm{EcN}$ at day 5 on different mannoside presenting surfaces. *EcN completely covered the BiPh-Man surface after 5 days of growth. The error bar represents the standard deviation of three parallel experiments.

Fig. 3. SEM images of the EcN biofilms formed after 120 hours of incubation on a BiPh-Man surface. (a) Lamellar and chunk extracelular polymeric substances (EPS) and stacks of EcN containing small apertures and holes. (b) Stacks of EcN showing about ten layers of bacterial cells. (c) Bacterial cells buried by EPS and connected by these EPS to form a cross-linked net structure. The SEM images were obtained with $15.00 \mathrm{~K} \times$ magnification in (a), $10.00 \mathrm{~K} \times$ magnification in (b) and $30.00 \mathrm{~K} \times$ magnification in (c), EHT = $15.00 \mathrm{kV}, \mathrm{WD}=13 \mathrm{~mm}$. 
Fig. 4. A reflected bright-field image at the middle of an $\mathrm{EcN}$ biofilm formed after 120 hours of incubation on a BiPh-Man surface. The z-scan images (step: $1.5 \mu \mathrm{m}$, range $\mathrm{z}_{0}: \pm 15.0 \mu \mathrm{m}$ ) on the right and bottom were obtained across the horizontal and vertical dot lines, respectively, indicating that the biofilm was approximately $15 \mu \mathrm{m}$ thick and fully covered the substrate surface.

Fig. 5. (a) Comparison of bacterial interference by $\mathrm{EcN}$ and fim + E. coli 83972 biofilms on various mannoside surfaces for 11 days against green fluorescent protein (GFP)-labelled E. faecalis. The areas of E. faecalis coverage on the non-fluorescent benign biofilms was measured from the fluorescence images, which represent a large underestimate for films with a high E. faecalis coverage due to the presence of E. faecalis outside the focal plane. The data for fim+ E. coli 83972 biofilms were taken from our previous report [6]. The data represent the mean of at least three experiments in which images were taken on 10 random locations on each surface. (b) The CFU counts of the $E$. faecalis on the various surfaces with/without EcN pre-treatment. Data were obtained in duplicate by detaching the bacteria from the surface with sonication followed by selective plating in the presence of tetracycline $(4 \mu \mathrm{g}$ $\mathrm{mL}^{-1}$ ). The values were expressed as mean +/- standard deviation. The density of E. faecalis adhered on the backside of the wafer was measured to

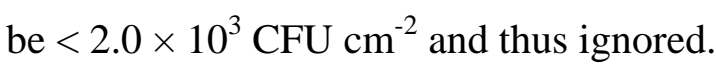

Fig. 6. Representative overlay of green fluorescence images (a)-(g) on reflected bright-field after bacterial interference against E. faecalis for 11 days using pre-formed biofilms of EcN. The substrates were incubated with $1 \mathrm{~mL} \mathrm{LB}$ broth containing $10^{8} \mathrm{CFU} \mathrm{mL}^{-1}\left([\mathrm{OD}]_{600}=0.25\right) \mathrm{EcN}$ for 5 days to allow the biofilm formation and then transferred to $1 \mathrm{~mL}^{\circ} 10^{8} \mathrm{CFU} \mathrm{mL} \mathrm{m}^{-1}\left([\mathrm{OD}]_{600}=\right.$ 0.25) E. faecalis culture in LB (culture replaced daily) for 11 days of incubation. The zoom-in image in (d) shows the GPF labelled E. faecalis bacteria marked by the white arrows. (h) Representative green fluorescence image of GFP labelled E. faecalis adhered on BiPh-Man surfaces (without 
pre-coating with $\mathrm{EcN}$ ) after 11 days.

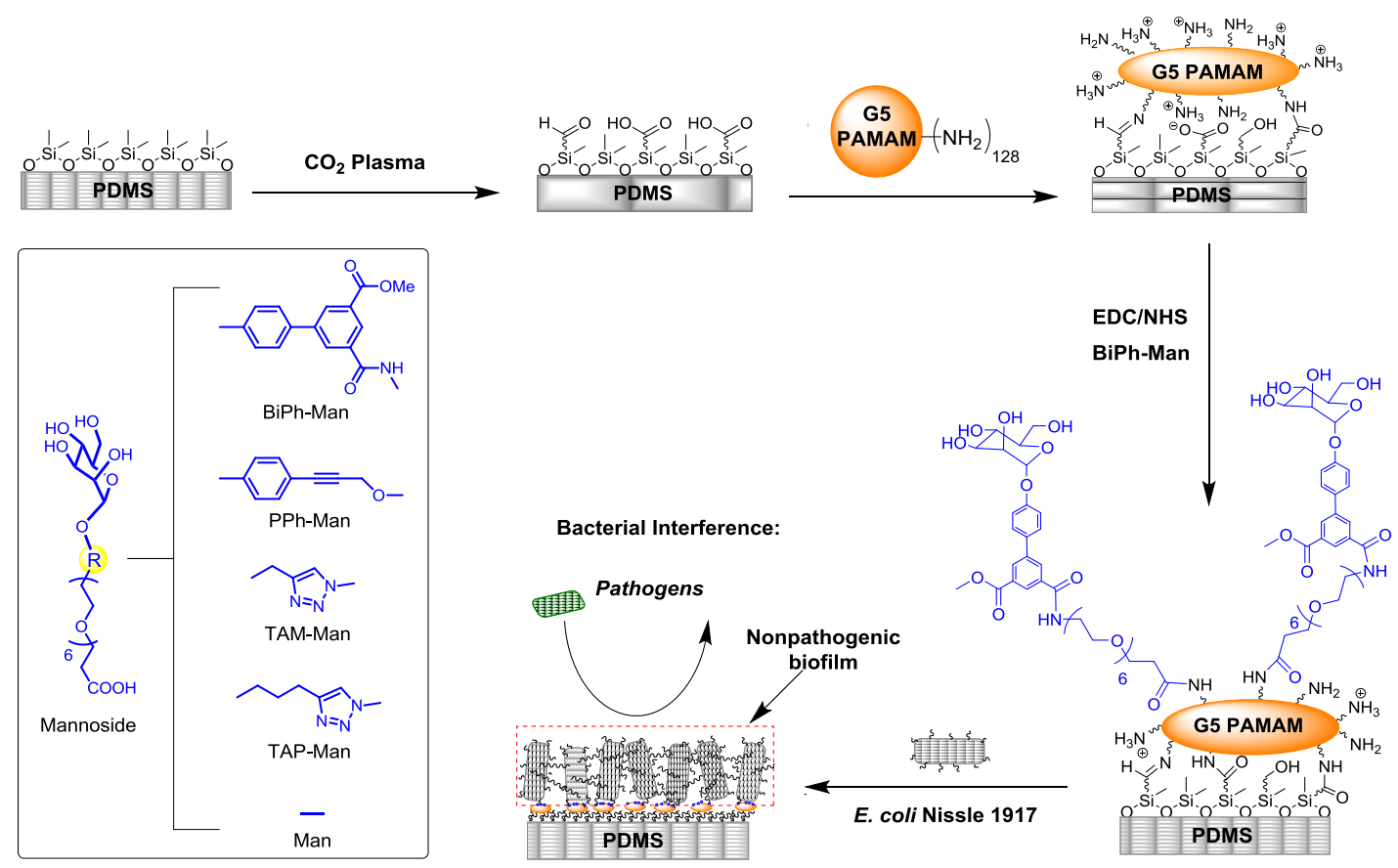

Scheme 1. Modification of PDMS with various mannosides for evaluation of their effectiveness in promoting the growth of non-pathogenic E. coli Nissle 1917 biofilms for bacterial interference against pathogenic colonization. The mannosides BiPh-Man, PPh-Man, TAM-Man, TAP-Man, and Man are differed by the glycosidic linkage R as shown in the box. 


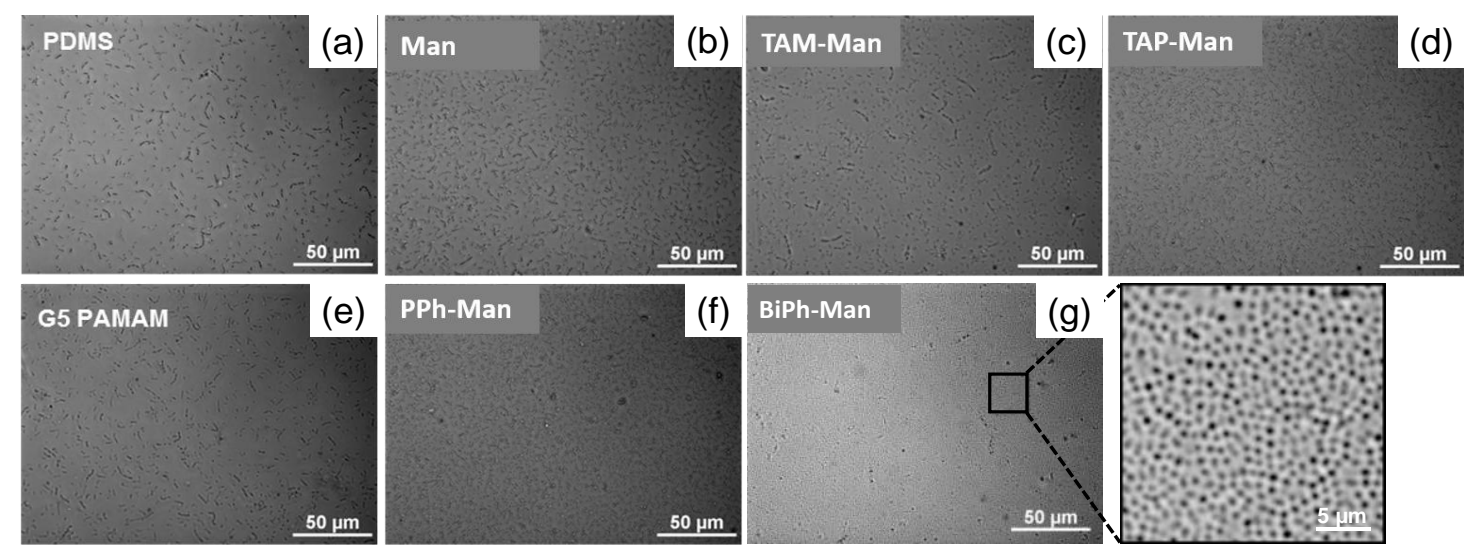

Fig. 1. Representative reflected bright-field images of $\mathrm{EcN}$ on control and mannoside-presenting PDMS surfaces after 5 days adhesion. Each surface was

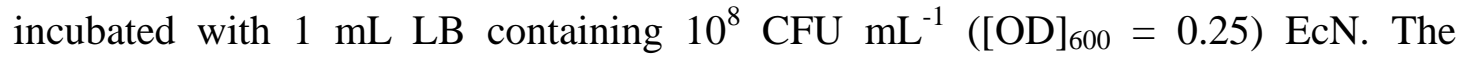
representative reflected bright-field image of $\mathrm{EcN}$ on BiPh-Man surface shows a complete coverage of the surface. In the zoom-in image, the dark spots on the relatively flat biofilm surface are presumably the entrance of channels $[4,5]$ for transporting nutrients and wastes in and out of the biofilm. Each image is representative of up to 10 images obtained on random locations at each surface and 3 experiments were repeated. 


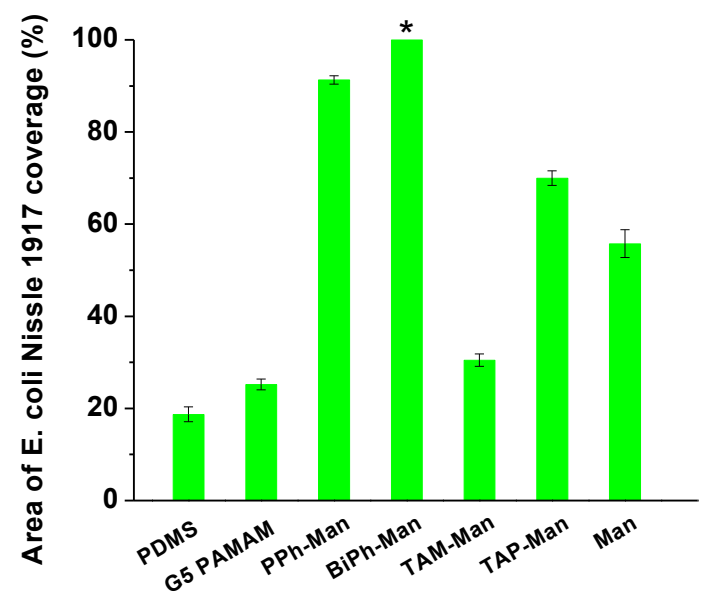

Fig. 2. Plot of the coverage of $\mathrm{EcN}$ at day 5 on different mannoside presenting surfaces. EcN completely covered the BiPh-Man surface after 5 days of growth. The data was the mean of a triplicate experiment and the error bar represents the standard deviation. 

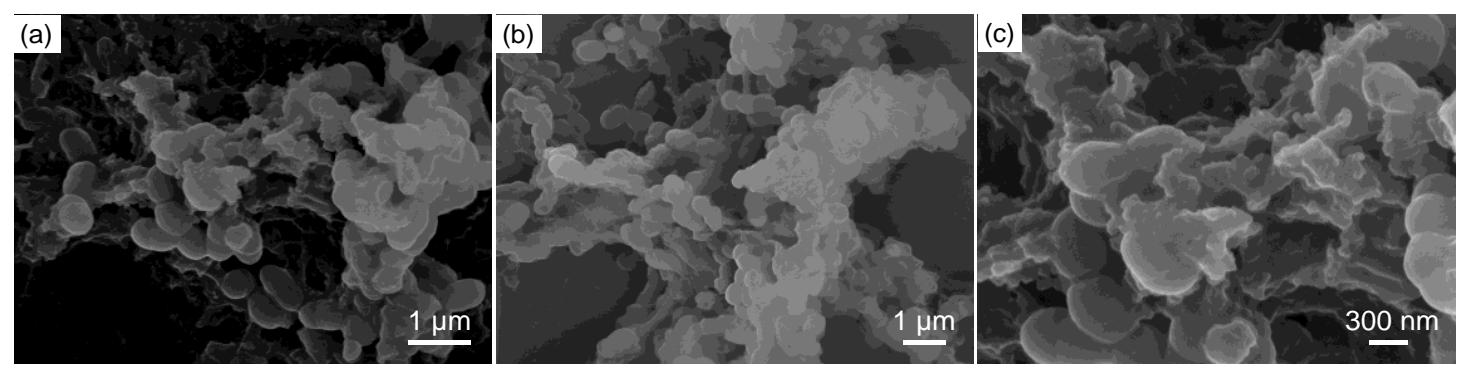

Fig. 3. SEM images of the EcN biofilms formed after 120 hours of incubation on a BiPh-Man surface. (a) Lamellar and chunk extracelular polymeric substances (EPS) and stacks of EcN containing small apertures and holes. (b) Stacks of EcN showing about ten layers of bacterial cells. (c) Bacterial cells buried by EPS and connected by these EPS to form a cross-linked net structure. The SEM images were obtained with $15.00 \mathrm{~K} \times$ magnification in (a), $10.00 \mathrm{~K} \times$ magnification in (b) and $30.00 \mathrm{~K} \times$ magnification in (c), EHT $=15.00 \mathrm{kV}, \mathrm{WD}=13 \mathrm{~mm}$. 


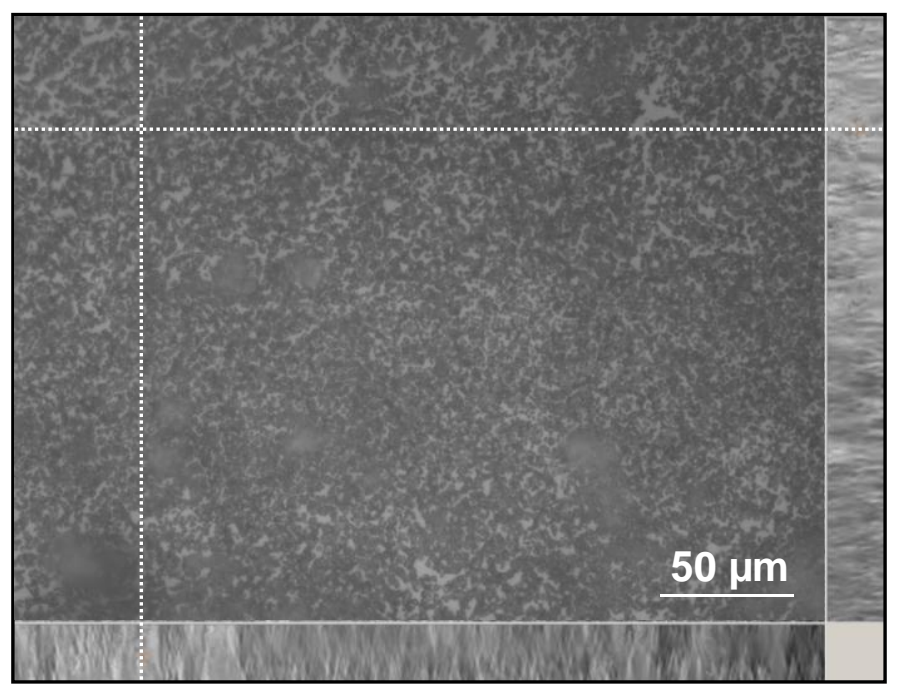

Fig. 4. A reflected bright-field image at the middle of an EcN biofilm formed after 120 hours of incubation on a BiPh-Man surface. The z-scan images (step: $1.5 \mu \mathrm{m}$, range $\left.\mathrm{z}_{0}: \pm 15.0 \mu \mathrm{m}\right)$ on the right and bottom were obtained across the horizontal and vertical dot lines, respectively, indicating that the biofilm was approximately $15 \mu \mathrm{m}$ thick and fully covered the substrate surface. 

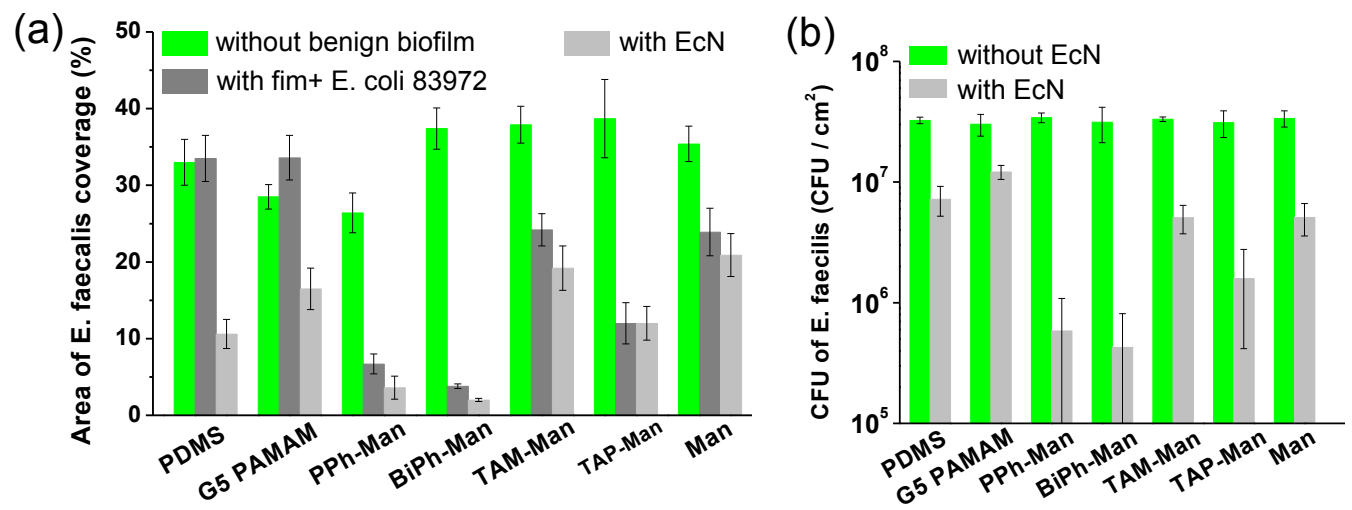

Fig. 5. (a) Comparison of bacterial interference by EcN and fim+ E. coli 83972 biofilms on various mannoside surfaces for 11 days against green fluorescent protein (GFP)-labelled E. faecalis. The areas of E. faecalis coverage on the non-fluorescent benign biofilms was measured from the fluorescence images, which represent a large underestimate for films with a high $E$. faecalis coverage due to the presence of $E$. faecalis outside the focal plane. The data for fim+ E. coli 83972 biofilms were taken from our previous report [6]. The data represent the mean of at least three experiments in which images were taken on 10 random locations on each surface. (b) The CFU counts of the E. faecalis on various surfaces with/without EcN pre-treatment. Data were obtained in duplicate by detaching the bacteria from the surface with sonication followed by selective plating in the presence of tetracycline $\left(4 \mu \mathrm{g} \mathrm{mL}^{-1}\right)$. The values were expressed as mean +/- standard deviation. The density of E. faecalis adhered on the backside of the wafer was measured to be $<2.0 \times 10^{3} \mathrm{CFU} \mathrm{cm}^{-2}$ and thus ignored. 


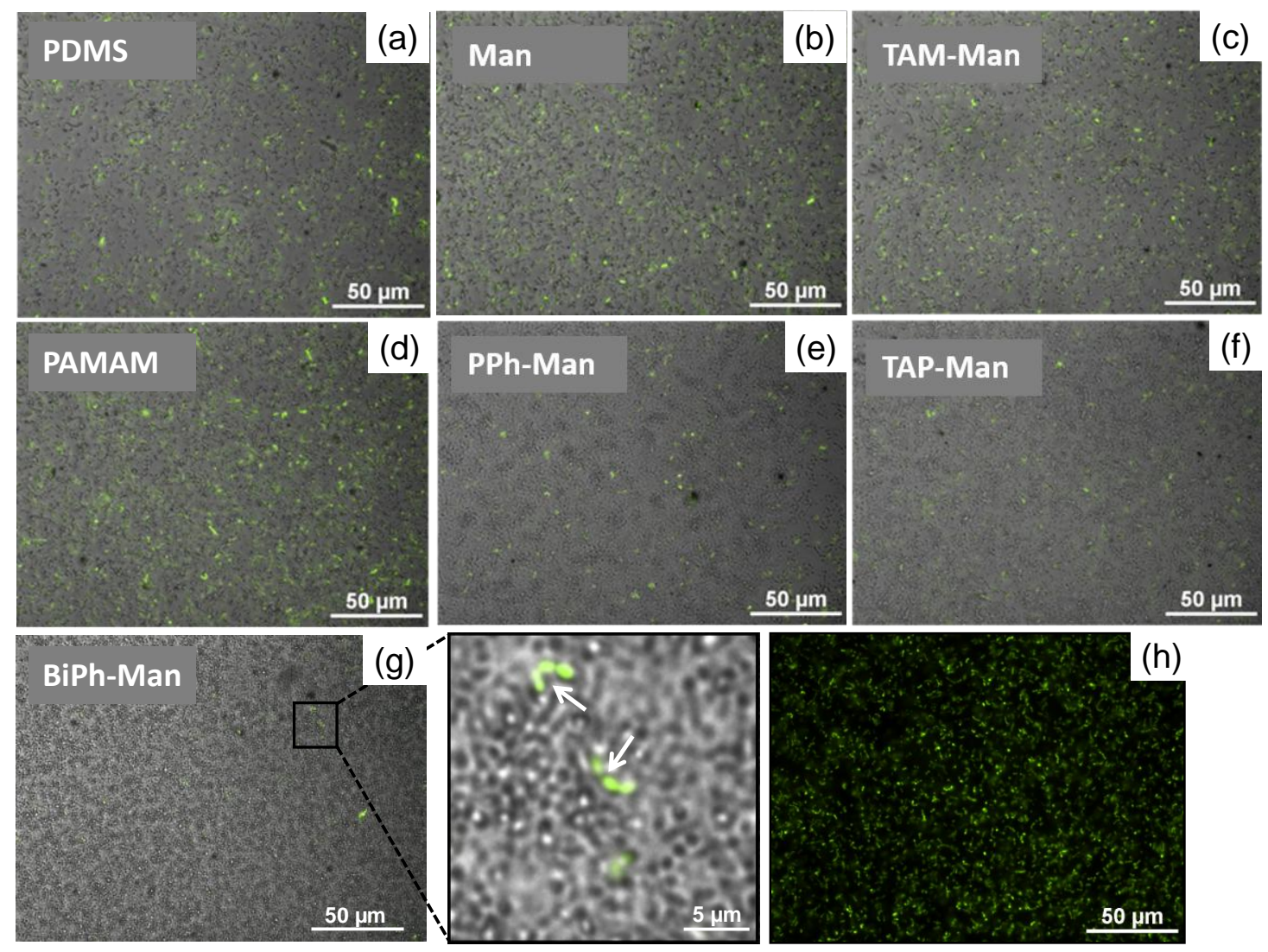

Fig. 6. Representative overlay of green fluorescence images (a)-(g) on reflected bright-field after bacterial interference against E. faecalis for 11 days using pre-formed biofilms of EcN. The substrates were incubated with $1 \mathrm{~mL} \mathrm{LB}$ broth containing $10^{8} \mathrm{CFU} \mathrm{mL} \mathrm{m}^{-1}\left([\mathrm{OD}]_{600}=0.25\right) \mathrm{EcN}$ for 5 days to allow the biofilm formation and then transferred to $1 \mathrm{~mL}$ of $10^{8} \mathrm{CFU} \mathrm{mL}^{-1}\left([\mathrm{OD}]_{600}=0.25\right)$ E. faecalis culture in LB (culture replaced daily) for 11 days of incubation. The zoom-in image in (d) shows the GPF labelled E. faecalis bacteria marked by the white arrows. (h) Representative green fluorescence image of GFP labelled E. faecalis adhered on BiPh-Man surfaces (without pre-coating with $\mathrm{EcN}$ ) after 11 days. 


\section{Graphical abstracts:}

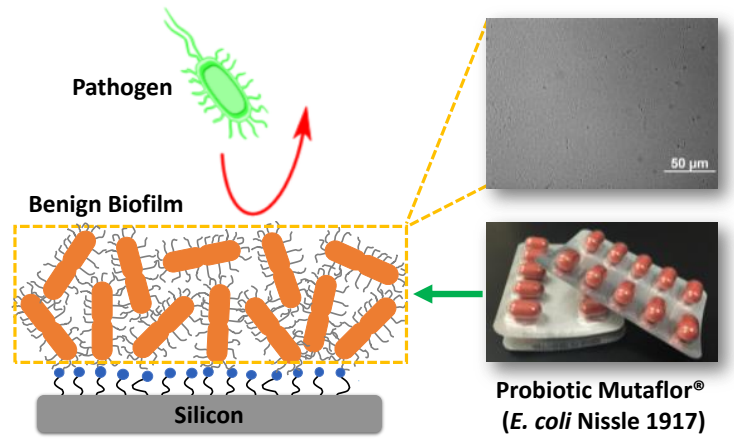

